PROCEEDINGS OF THE

AMERICAN MATHEMATICAL SOCIETY

Volume 126, Number 8, August 1998, Pages 2453-2458

S 0002-9939(98)04289-0

\title{
RANK ONE LATTICES WHOSE PARABOLIC ISOMETRIES HAVE NO ROTATIONAL PART
}

\author{
CHRISTOPH HUMMEL \\ (Communicated by Christopher Croke)
}

\begin{abstract}
We prove a result on certain finite index subgroups of rank one lattices which is motivated by cusp closing constructions.
\end{abstract}

Let $X$ always denote a rank one symmetric space of non-compact type, i.e., $X$ is the hyperbolic space $\mathbb{K} H^{n}, n \geq 2$, where $\mathbb{K}$ is either $\mathbb{R}, \mathbb{C}, \mathbb{H}$ or $\mathbb{O}$ and $n=2$ in the latter case. By $\Sigma$ we always denote a lattice in the isometry group $\operatorname{Iso}(X)$, that is, a discrete subgroup of the isometry group $\operatorname{Iso}(X)$ of $X$ such that the quotient $\Sigma \backslash X$ has finite volume. The result below is only relevant for non-uniform lattices, i.e. lattices $\Sigma$ where $\Sigma \backslash X$ is non-compact. Such lattices exist for each $X$ by a result of A. Borel (see [B1] or Chapter XIV in [R]).

We say that a parabolic isometry $\sigma \in \operatorname{Iso}(X)$ has no rotational part if $\sigma$ is contained in the nilpotent part $N$ of some Iwasawa decomposition $\operatorname{Iso}^{0}(X)=N A K$. Here $\operatorname{Iso}^{0}(X)$ denotes the identity component of $\operatorname{Iso}(X)$. In this note we give a simple geometric argument in order to prove the theorem below, which is related to a result of Borel and Garland \& Raghunathan.

Theorem. For any lattice $\Sigma<\operatorname{Iso}(X)$ in the isometry group of a rank one symmetric space $X$ of non-compact type there exists a finite subset $F \subset \Sigma$ of parabolic isometries such that the following holds. Assume $\Sigma^{\prime} \triangleleft \Sigma$ is a normal subgroup and $\Sigma^{\prime} \cap F=\emptyset$. Then any parabolic isometry in $\Sigma^{\prime}$ has no rotational part.

Remark. The proof of the theorem provides an explicit procedure to determine $F$, which is in some sense optimal by the example at the end.

As a consequence of the theorem we obtain the following corollary. The statement is proved in [GR], Lemma 6.5, with Proposition 17.6 from [B2], and the methods are of algebraic nature.

Corollary (Borel and Garland \& Raghunathan). Let $V=\Sigma \backslash X$ be a complete, locally rank one symmetric manifold of non-compact type and finite volume, and denote by $N$ the nilpotent part of an Iwasawa decomposition of $\operatorname{Iso}^{0}(X)$. Then there exists a finite regular covering $\widehat{V} \rightarrow V$ such that each cusp of $\widehat{V}$ is diffeomorphic to $\Gamma \backslash N \times[0, \infty)$ for some lattice $\Gamma<N$.

Received by the editors December 7, 1996 and, in revised form, January 22, 1997.

1991 Mathematics Subject Classification. Primary 53C35; Secondary 22E40, 22E25.

Key words and phrases. Rank one lattices, rotational part, cusp closing.

The author is supported by the Swiss National Science Foundation.

(C) 1998 American Mathematical Society 
Remarks. 1. Another proof of this corollary, in the case when $X$ is real hyperbolic, can be found in $[\mathrm{AF}]$.

2. Using Borel's existence result for non-uniform lattices (refer to Chapter XIV in $[R]$ ), this corollary guarantees the existence of locally real and complex hyperbolic spaces in each dimension, whose cusps can be closed as manifolds of non-positive curvature (see $[\mathrm{S}],[\mathrm{BK}]$, $[\mathrm{HS}]$ and $[\mathrm{H}]$ ). Cusp closing is one of the few methods currently known to construct closed manifolds of nonpositive sectional curvature and rank 1 containing 2 -flats. Hence we think it is of interest to have an elementary geometric proof of this result.

\section{PRELIMINARIES}

In this section we briefly recall the structure of the ends of finite volume quotients of rank one symmetric spaces of non-compact type, and fix the notation. Basic references for this section are [E1], [E2] and [K].

Let $X$ and $\Sigma$ be as above. Then $\Sigma \backslash X$ has finitely many pairwise disjoint cusps $\mathcal{C}_{1}, \ldots, \mathcal{C}_{m} \subset \Sigma \backslash X$ such that

$$
\Sigma \backslash X-\bigcup_{i=1}^{m} \mathcal{C}_{i} \subset \Sigma \backslash X
$$

is relatively compact (see Lemma 6.6 in $[\mathrm{GR}]$ ). The cusps $\mathcal{C}_{1}, \ldots, \mathcal{C}_{m}$ are in one-toone correspondence with the conjugacy classes of the maximal parabolic subgroups of $\Sigma$. One can describe the cusps and their relation to maximal parabolic subgroups of $\Sigma$ as follows.

For each cusp $\mathcal{C} \subset X$ there is a maximal parabolic subgroup $\Pi<\Sigma$, uniquely determined up to conjugacy in $\Sigma$, and a closed horoball $\mathrm{HB} \subset X$ stabilized by $\Pi$ so that $\Pi \backslash$ HB naturally identifies with $\mathcal{C}$. After choosing an Iwasawa decomposition Iso $^{0}(X)=N A K$ such that HB is stabilized by $N$, one gets a natural identification $\partial \mathrm{HB} \simeq N$ of the horosphere $\partial \mathrm{HB}$ with the simply connected nilpotent Lie group $N$. In this way $N$ inherits a left invariant metric, and one may view $\Pi$ as a lattice in the isometry group Iso $(N)$. The cusp $\Pi \backslash$ HB is diffeomorphic to $\Pi \backslash N \times[0, \infty)$.

The Lie group $N$ satisfies $[N,[N, N]]=\{e\}$, where $e$ denotes the identity of $N$. Hence the simply connected Lie group $Z:=[N, N]$ is abelian and contained in the center of $N$. We remark that $N$ is abelian for $\mathbb{K}=\mathbb{R}$ and of Heisenberg type with $\operatorname{dim} Z=\operatorname{dim}(\operatorname{Im} \mathbb{K})=1,3$ and 7 for $\mathbb{K}=\mathbb{C}, \mathbb{H}$ and $\mathbb{O}$, respectively.

Denote by $\mathfrak{n}$ and $\mathfrak{z}$ the Lie algebra of $N$ and $Z$, respectively, and view $\mathfrak{z}$ as a subalgebra of $\mathfrak{n}$. By exp: $\mathfrak{n} \rightarrow N$ we mean the Lie group exponential map, which is a diffeomorphism since $N$ is nilpotent and simply connected. The Campbell-BakerHausdorff formula reduces to

$$
\exp (\xi) \exp (\eta)=\exp \left(\xi+\eta+\frac{1}{2}[\xi, \eta]\right)
$$

and holds for any $\xi, \eta \in \mathfrak{n}$. This implies that $[\exp (\xi), \exp (\eta)]=\exp ([\xi, \eta])$ for each $\xi, \eta \in \mathfrak{n}$. Now it is easy to see that for each $g \in N-Z$ the map $[g, \cdot]: N \rightarrow Z$ is surjective (use [E2], Sect. 1).

If $\Gamma<N$ is a lattice, then $\Gamma \backslash N$ is compact and there is a basis $\mathcal{T}$ of the underlying vector space of $\mathfrak{n}$ such that $\exp (\mathcal{T}) \subset \Gamma$ and $\mathcal{T} \cap \mathfrak{z}$ generates $\mathfrak{z}$.

We denote by $\operatorname{Aut}(N)$ the automorphism group of the Lie group $N$ and put

$$
R:=\operatorname{Iso}(N) \cap \operatorname{Aut}(N) .
$$


Then $\operatorname{Iso}(N)$ is canonically isomorphic to the semi-direct product $\operatorname{Iso}(N)=N \rtimes R$ acting on $N$ by

$$
(a, A)(g)=a \cdot A(g)
$$

for $(a, A) \in N \rtimes R$ and $g \in N$. For an element in $N \rtimes R$, the component in the $R$-factor is called its rotational part. An isometry of $N$ is called rotation free or equivalently a translation if its rotational part is trivial, i.e., it is contained in $N \rtimes\{\mathbf{1}\} \simeq N$.

Note that, by definition of $R$, the differential $d A: \mathfrak{n} \rightarrow \mathfrak{n}$ of any $A \in R$ respects the orthogonal decomposition $\mathfrak{n}=\mathfrak{z}^{\perp} \oplus \mathfrak{z}$ of the underlying vector space.

For any $\phi \in \operatorname{Iso}(N)$ we define $|\phi|$ to be the distance in $N$ between $e$ and $\phi(e)$ with respect to the left invariant metric distinguished above. Observe that $|\phi \psi| \leq$ $|\phi|+|\psi|$ for any $\phi, \psi \in \operatorname{Iso}(N)$. By $\bar{B}_{r}(g):=\left\{h \in N|| g^{-1} h \mid \leq r\right\}$ we denote the closed distance ball in $N$ of radius $r$ centered at $g \in N$.

\section{Proof OF THE RESULT}

The theorem is an easy consequence of the following

Lemma. For any lattice $\Gamma<\operatorname{Iso}(N)$ there exists a constant $C \geq 0$ such that the following is true. Assume $\Gamma^{\prime} \triangleleft \Gamma$ with $\left|\gamma^{\prime}\right|>C$ for each $\gamma^{\prime} \in \Gamma^{\prime}-\{e\}$. Then $\Gamma^{\prime}<\Gamma \cap N$.

Remark. Given $\Gamma$, one can explicitly determine a constant $C$ satisfying the lemma from the group of translations $\Gamma \cap N$, as the following proof shows.

Proof. Auslander's generalized Bieberbach theorem [A] states that for any lattice $\Gamma<N \rtimes R$ the normal subgroup of translations $\Gamma \cap N \triangleleft \Gamma$ has finite index in $\Gamma$. Hence we can choose a basis $\mathcal{T} \subset \mathfrak{n}$ of $\mathfrak{n}$ such that $\mathcal{T} \cap \mathfrak{z}$ generates $\mathfrak{z}$ and $\exp (\mathcal{T}) \subset \Gamma \cap N$. Then we pick some $\delta>0$ such that

$$
\bar{B}_{\delta}(h) \cap(\Gamma \cap N) \neq \emptyset \quad \text { and } \quad|\exp (\tau)| \leq \delta
$$

for each $h \in N$ and each $\tau \in \mathcal{T}$. Finally, we choose $\epsilon>0$ such that

$$
\left[\bar{B}_{2 \delta}(e), \bar{B}_{\delta}(e)\right] \subset \bar{B}_{\epsilon}(e)
$$

and put

$$
C:=\epsilon+2 \delta \text {. }
$$

Now assume $\Gamma^{\prime} \triangleleft \Gamma$ with $\left|\gamma^{\prime}\right|>C$ for each $\gamma^{\prime} \in \Gamma^{\prime}-\{e\}$, and pick any $(a, A) \in \Gamma^{\prime}$. We have to show that $A=\mathbf{1}$. Note that $t \cdot(a, A)=(t a, A) \in \Gamma$ for any $t \in \Gamma \cap N$, and thus $[(t a, A),(a, A)] \in \Gamma^{\prime}$ since $\Gamma^{\prime}$ is a normal subgroup of $\Gamma$. We compute

$$
[(t a, A),(a, A)]=\left(t a A\left(t^{-1}\right) a^{-1}, \mathbf{1}\right)=t A\left(t^{-1}\right)[A(t), a]=t A\left(t^{-1}\right) z \in \Gamma^{\prime},
$$

where $z=[A(t), a] \in Z$.

First assume $t \in Z$. Then we see that $z=e$, and thus $t A\left(t^{-1}\right) \in \Gamma^{\prime} \cap Z$. If $t \in \exp (\mathcal{T}) \cap Z$, then $\left|t A\left(t^{-1}\right)\right| \leq|t|+\left|A\left(t^{-1}\right)\right| \leq 2 \delta$, and hence $t A\left(t^{-1}\right)=e$ by the assumption on $\Gamma^{\prime}$. Since $\exp (\mathcal{T}) \cap Z$ generates $Z$, this implies that $\left.A\right|_{Z}=\operatorname{id}_{Z}$.

Remark. Observe that the same argument already proves the lemma in case $N$ is abelian. Moreover, in this case we can even take $C=2 \delta$. 
In general, it remains to show that $\left.d A\right|_{\mathfrak{z}^{\perp}}=\mathrm{id}_{\mathfrak{z}^{\perp}}$. We assume $|t| \leq \delta$ and we abbreviate $g:=t A\left(t^{-1}\right)$. Since $z g \in \Gamma^{\prime} \triangleleft \Gamma$, we conclude that for any $v \in \Gamma \cap N$ we have $v z g v^{-1}=z v g v^{-1}=[v, g] z g \in \Gamma^{\prime}$. We claim that

$$
g=t A\left(t^{-1}\right) \in Z \text {. }
$$

Assuming that $g \notin Z$, there exists some $h \in N$ such that $[g, h]=z$. By the choice of $\delta$, we find some $v \in \Gamma \cap N$ such that $\left|v^{-1} h\right| \leq \delta$, and we compute

$$
\begin{aligned}
\Gamma^{\prime} \ni[v, g] z g & =[v, g][g, h] g=v g v^{-1} h g^{-1} h^{-1} g \\
& =v\left[g, v^{-1} h\right] v^{-1} h g g^{-1} h^{-1} g=\left[g, v^{-1} h\right] g .
\end{aligned}
$$

We see from the choice of $\epsilon$ that $\left|\left[g, v^{-1} h\right]\right| \leq \epsilon$, and hence $\left|\left[g, v^{-1} h\right] g\right| \leq \epsilon+2 \delta=C$ since $|g| \leq 2 \delta$. From the assumption on $\Gamma^{\prime}$, we obtain that $\left[g, v^{-1} h\right] g=e$ and thus $g=t A\left(t^{-1}\right) \in Z$.

Pick $\tau \in \mathcal{T}$, and let $\eta \in \mathfrak{z}^{\perp}$ and $\zeta \in \mathfrak{z}$ be the components of $\tau \in \mathcal{T}$ with respect to the vector space decomposition $\mathfrak{n}=\mathfrak{z}^{\perp} \oplus \mathfrak{z}$. We conclude that

$$
\begin{aligned}
Z \ni \exp (\tau) A(\exp (-\tau)) & =\exp (\tau) \exp (-d A(\tau)) \\
& =\exp (\eta+\zeta) \exp \left(-\left.d A\right|_{\mathfrak{z}^{\perp}}(\eta)-\left.d A\right|_{\mathfrak{z}}(\zeta)\right) \\
& =\exp \left(\eta-\left.d A\right|_{\mathfrak{z}^{\perp}}(\eta)-\frac{1}{2}\left[\eta,\left.d A\right|_{\mathfrak{z}^{\perp}}(\eta)\right]\right) .
\end{aligned}
$$

The right hand side is contained in $Z$ if and only if $\left.d A\right|_{\mathfrak{z}^{\perp}}(\eta)=\eta$. Since $\mathcal{T}$ is a basis of $\mathfrak{n}$, we obtain $\left.d A\right|_{\mathfrak{z}^{\perp}}=\mathrm{id}_{\mathfrak{z}^{\perp}}$.

We now conclude the proof of the theorem. So assume $\Sigma$ is a lattice in $X$. Let $\Pi_{1}, \ldots, \Pi_{m}$ be finitely many maximal parabolic subgroups of $\Sigma$ such that each maximal parabolic subgroup of $\Sigma$ is conjugate to some $\Pi_{i}$. For each $\Pi_{i}$ choose $C_{i} \geq 0$ such that the lemma holds with $\Gamma=\Pi_{i}$ and $C=C_{i}$. Then the set

$$
F:=\bigcup_{i=1}^{m}\left\{\sigma_{i} \in \Pi_{i}|0<| \sigma_{i} \mid \leq C_{i}\right\}
$$

has the desired properties.

Indeed, suppose $\Sigma^{\prime} \triangleleft \Sigma$ is such that $\Sigma^{\prime} \cap F=\emptyset$. Let $\sigma^{\prime} \in \Sigma^{\prime}$ be a parabolic isometry. We have to show that $\sigma^{\prime}$ has no rotational part. Therefore observe that $\sigma^{\prime}$ has no rotational part if and only if some conjugate of $\sigma^{\prime}$ in $\operatorname{Iso}(X)$ has no rotational part. By the choice of $\Pi_{1}, \ldots, \Pi_{m}$ there is some $i$ and some $\sigma \in \Pi_{i}$ conjugate to $\sigma^{\prime}$ in $\Sigma$. Since $\Sigma^{\prime} \triangleleft \Sigma$, we have $\sigma \in \Pi_{i} \cap \Sigma^{\prime} \triangleleft \Pi_{i}$. Hence $\sigma$, and therefore also $\sigma^{\prime}$, has no rotational part by the choice of $F$. This finishes the proof of the theorem.

Proof of the Corollary. Let $V=\Sigma \backslash X$ be as in the corollary. We choose a finite subset $F \subset \Sigma$ of parabolic isometries for the rank one lattice $\Sigma$ according to the theorem. Since $\Sigma$ is finitely generated (see Theorem 0.9 in [GR]), it is residually finite by a theorem of Malcev (see Theorem 4.2 in [W]). Hence there exists a normal subgroup $\widehat{\Sigma} \triangleleft \Sigma$ of finite index satisfying $\widehat{\Sigma} \cap F=\emptyset$.

The natural map $\widehat{V}: \widehat{\Sigma} \backslash X \rightarrow \Sigma \backslash X=V$ is a finite, regular covering. By the choice of $F$, each parabolic isometry in $\widehat{\Sigma}$ has no rotational part, and thus the cusps of $\widehat{V}$ are of the requested type.

Example. As a final remark we give a simple example which shows that the construction in the proof of the lemma is optimal in case $N$ is abelian. Denote by 
$e_{1}, e_{2}$ the standard orthonormal basis of the Euclidean plane $\mathbb{R}^{2}$ and let $S$ be the reflection at the line $\mathbb{R} e_{1}$, i.e., $S\left(e_{1}\right)=e_{1}$ and $S\left(e_{2}\right)=-e_{2}$. For any integer $k$, the elements

$$
\left(\left(k+\frac{1}{2}\right) e_{1}, S\right),\left(2 e_{2}, \mathbf{1}\right) \in \mathbb{R}^{2} \rtimes \mathrm{O}(2) \simeq \operatorname{Iso}\left(\mathbb{R}^{2}\right)
$$

generate a lattice $\Gamma_{k}$ in Iso $\left(\mathbb{R}^{2}\right)$, namely

$$
\Gamma_{k}=\left\{\left(\frac{m(2 k+1)}{2} e_{1}+2 n e_{2}, S^{m}\right) \mid n, m \in \mathbb{Z}\right\},
$$

and the quotients $\Gamma_{k} \backslash \mathbb{R}^{2}$ are Klein bottles. In particular, $\Gamma_{k}$ is not a group of translations. Let $\Gamma_{*}$ be the lattice in $\operatorname{Iso}\left(\mathbb{R}^{2}\right)$ generated by

$$
\left(\frac{1}{2} e_{1}, S\right),\left(e_{2}, \mathbf{1}\right) \in \mathbb{R}^{2} \rtimes \mathrm{O}(2) .
$$

It is readily verified that each $\Gamma_{k}$ is a normal subgroup of $\Gamma_{*}$. Observe further that $|\gamma| \geq 2$ for each non-trivial isometry $\gamma \in \Gamma_{k}$ and any $k \geq 2$. The proof of the lemma shows that any normal subgroup $\Gamma^{\prime} \triangleleft \Gamma_{*}$ with $\left|\gamma^{\prime}\right|>2$ for each $\gamma \in \Gamma^{\prime}-\{0\}$ is a group of translations. Indeed, we observe that $\Gamma_{*} \cap \mathbb{R}^{2}=\mathbb{Z}^{2}$. Consequently and in view of the remark above, the proof of the lemma works for $\Gamma=\Gamma_{*}$ with $\exp (\mathcal{T})=\left\{e_{1}, e_{2}\right\}, \delta=1$ and hence $C=2$.

This note is taken from my thesis [H], and I wish to thank my advisor Viktor Schroeder and also Enrico Leuzinger for valuable discussions and suggestions. For useful comments I thank the referee. I am grateful to the Department of Mathematics at the University of Pennsylvania for its hospitality, and to the Swiss National Science Foundation for financial support.

\section{REFERENCES}

[A] L. Auslander, Bieberbach's Theorems on Space Groups and Discrete Uniform Subgroups of Lie Groups, Ann. of Math. 71 (1960), 579-589. MR 22:12161

[AF] C.S. Aravinda, F.T. Farrell, Rank 1 Aspherical manifolds which do not support any nonpositively curved metric, Comm. Anal. Geom. 2 (1994), 65-78. MR 96e:53045

[B1] A. Borel, Compact Clifford-Klein forms of symmetric spaces, Topology 2 (1963), 111-122. MR 26:3823

[B2] A. Borel, Introduction aux groupes arithmétiques, Hermann, Paris, 1969. MR 39:5577

[BK] S.V. Buyalo, V.L. Kobel'skiı̌, Cusp Closing of Hyperbolic Manifolds, Geometriae Dedicata 59 (1996), 147-156. MR 97g:53043

[E1] P. Eberlein, Lattices in spaces of nonpositive curvature, Ann. Math. 111 (1980), 435-476. MR 82m:53040

[E2] P. Eberlein, Geometry of 2-step nilpotent groups with a left invariant metric, Ann. Sci École Norm. Sup. 27 (1994), 611-660. MR 95m:53059

[GR] H. Garland, M.S. Raghunathan, Fundamental domains in (RR-)rank 1 semisimple Lie groups, Ann. Math. 92 (1970), 279-326. MR 42:1943

[H] C. Hummel, Closing Complex Hyperbolic Cusps and Applications, Dissertation, Universität Zürich, 1996.

[HS] C. Hummel, V. Schroeder, Cusp Closing in Rank One Symmetric Spaces, Invent. math. 123 (1996), 283-307. MR 97e:53098

[K] A. Kaplan, Riemannian Nilmanifolds attached to Clifford Modules, Geometriae Dedicata 11 (1981), 127-136. MR 82h:22008

[R] M.S. Raghunathan, Discrete Subgroups of Lie Groups, Springer, Berlin Heidelberg New York, 1972. MR 58:22394a 
[S] V. Schroeder, A Cusp Closing Theorem, Proc. Amer. Math. Soc. 106 (1989), 797-802. MR 89k:53045

[W] B.A.F. Wehrfritz, Infinite Linear Groups, Springer, Berlin Heidelberg New York, 1973. MR 49:436

Department of Mathematics, University of Pennsylvania, 209 South $33^{\text {RD }}$ Street, Philadelphia, Pennsylvania 19104

E-mail address: hummelc@math.upenn.edu

Current address: Departement Mathematik, ETH-Zentrum, CH-8092 Zürich, Switzerland

E-mail address: hummel@math.ethz.ch 